A Novel Tmem119-tdTomato Reporter Mouse Model for Studying Microglia in the

\title{
Central Nervous System
}

3 Chunsheng Ruan ${ }^{\mathrm{a}}$, Linlin Sun ${ }^{\mathrm{b}}$, Alexandra Kroshilina ${ }^{\mathrm{a}}$, Lien Beckers ${ }^{\mathrm{a}}$, Philip L. De Jager ${ }^{\mathrm{a}}$,

4 Elizabeth M. Bradshaw ${ }^{\mathrm{a}}$, Samuel Hasson ${ }^{\mathrm{c}, \mathrm{d}}$, Guang Yang ${ }^{\mathrm{b}}$, and Wassim Elyaman ${ }^{\mathrm{a}, *}$

6 a Center for Translational and Computational Neuroimmunology, Department of Neurology,

7 Columbia University Medical Center, New York, NY 10032, USA

$8{ }^{\mathrm{b}}$ Department of Anesthesiology, Columbia University Medical Center, New York, NY

9 10032, USA

$10{ }^{\mathrm{c}}$ Pfizer Inc., Cambridge, MA 02141, USA

11 dAmgen, Cambridge, MA 02141, USA

12

13 *Corresponding author at: Center for Translational and Computational Neuroimmunology,

14 Department of Neurology, Columbia University Medical Center, New York, NY 10032, USA.

15 Email address: we2152@cumc.columbia.edu (W. Elyaman) 


\section{Abstract}

17 Microglia are resident immune cells of the central nervous system (CNS). The exact role of

18 microglia in the physiopathology of CNS disorders is not clear due to lack of tools to

19 discriminate between CNS resident and infiltrated innate immune cells. Here, we present a

20 novel reporter mouse model targeting a microglia-specific marker (TMEM119) for studying

21 the function of microglia in health and disease. By placing a reporter cassette (GSG-3xFlag-

22 P2A-tdTomato) between the coding sequence of exon 2 and 3'UTR of the Tmem119 gene

23 using CRISPR/Cas9 technology, we generated a Tmem119-tdTomato knock-in mouse strain.

24 Gene expression assay showed no difference of endogenous Tmem119 mRNA level in the

25 CNS of Tmem119 $9^{\text {tdTomato/+ }}$ relative to control Wild-type mice. The cells expressing tdTomato-

26 were recognized by immunofluorescence staining using commercially available anti-

27 TMEM119 antibodies. Using immunofluorescence and flow cytometry techniques,

28 tdTomato $^{+}$cells were detected throughout the CNS, but not in peripheral tissues of adult

29 Tmem $119^{\text {tdTomato/+ }}$ mice. In addition, aging does not seem to influence TMEM119 expression

30 as tdTomato $^{+}$cells were detectable in the CNS of older mice (300 and 540 days old). Further

31 immunofluorescence characterization shows that the tdTomato ${ }^{+}$cells were highly colocalized

32 with $\mathrm{Iba1}^{+}$cells (microglia and macrophages) in the brain, but not with NeuN- (neurons),

33 GFAP- (astrocytes) or Olig2- (oligodendrocytes) labeled cells. Moreover, flow cytometry

34 analysis of brain tissues of adult mice demonstrates that the majority of microglial

$35 \mathrm{CD} 45^{\text {low }} \mathrm{CD} 11 \mathrm{~b}^{+}$cells $(96.6 \%)$ are tdTomato positive. Functionally, using a laser-induced

36 injury model, we measured time-lapse activation of tdTomato-labeled microglia by

37 transcranial two-photon microscopy in live Tmem119 $19^{\text {tdTomato/+ }}$ mice. Taken together, the

38 Tmem119-tdTomato reporter mouse model will serve as a valuable tool to specifically study

39 the role of microglia in health and disease. 


\section{Introduction}

Microglia constitute the resident macrophages of the central nervous system (CNS)(Graeber and Streit, 1990), with a panoply of functions including immune surveillance (Hanisch and Kettenmann, 2007), maintenance of neuronal networks (Wu et al., 2015), and injury repair (Colonna and Butovsky, 2017). Microglia are phagocytic cells, and clearance of debris is thought to be a key function, especially in aging (Pluvinage et al., 2019). They can also secrete a wide range of cytokines including chemokines. Historically, microglia were often described as resting (i.e., ramified), but this phrasing failed to convey the dynamic remodeling of their fine processes and constitutive immunosurveillance activity, and are now termed homeostatic (Boche et al., 2013; Lawson et al., 1993). This morphology was in contrast to a more amoeboid phenotype microglia can also obtain, which was referred to as macrophages, but now is more commonly referred to as activated to avoid confusion with infiltrating cells (Arcuri et al., 2017). Both morphological and physiological properties have been used to identify microglial cells. The markers frequently used to identify microglia are CD68, MHC class II, CD11b or Iba1. However, since these markers are also expressed on other myeloid cells, they fail to distinguish between resident microglia and infiltrating macrophages.

The importance of microglia in most neurodegenerative diseases, such as multiple sclerosis and Alzheimer's disease, is increasingly appreciated (Pena-Altamira et al., 2016; Wang and Colonna, 2019). As microglia are highly plastic, the local disease environment, where microglia are in close interaction with other CNS cell types, differs between different pathologies and different regions leading to various phenotypes. Therefore, the microglial phenotype is disease-dependent, influenced by neighboring cell types and disease pathologies such as aggregated amyloid-beta (Bachiller et al., 2018). Neurodegenerative diseases such as multiple sclerosis are associated with infiltration of peripheral monocytes/macrophages that 
65 contribute to disease pathogenesis (Meyer-Luehmann and Prinz, 2015). Many markers used

66 for the identification of microglia are also present in macrophages, as both cell types are

67 myeloid and share many transcriptional programs. Given a lack of reliable markers that can

68 distinguish between resident microglia and infiltrating myeloid cells, the specific function of

69 microglia in CNS diseases has been difficult to ascertain.

70 During the past few years, our group and others have generated bulk and single-cell

71 transcriptomics data to identify specific markers of human and mouse microglia (Bachiller et

72 al., 2018; Bennett et al., 2016; Butovsky et al., 2014; Chiu et al., 2013; Fahrenhold et al.,

73 2018; Gosselin et al., 2017; Hickman et al., 2013; Katsumoto et al., 2014; Konishi et al.,

74 2017; Marta Olah, 2019; Olah et al., 2018). Bennett et al identified Transmembrane protein

75119 (TMEM119) as a specific marker of human and mouse microglia and generated an anti-

76 TMEM119 monoclonal antibody to detect microglia in situ (Bennett et al., 2016).

77 TMEM119, an evolutionarily conserved 58-kDa type 1 transmembrane protein, was

78 originally identified as a regulator of osteoblast differentiation. It is also expressed on human

79 osteoblasts and follicular dendritic cells. Mice homozygous for a targeted deletion of the

80 Tmem119 gene exhibit growth retardation associated with delayed endochondral bone

81 ossification and impaired osteoblast differentiation(Hisa et al., 2011). Although the role of

82 TMEM119 in bone development is becoming clear, whether TMEM119 is involved in

83 microglia development and function is not known.

84 In an effort to develop a reliable tool for studying microglia, we have generated a novel

85 microglia reporter mouse strain where the tdTomato fluorescence gene was knocked-in

86 between the coding sequence of exon 2 and the termination codon in the 3' UTR of the

87 Tmem119 gene thus preserving endogenous Tmem119 expression. Using

88 immunofluorescence and flow cytometry analyses, we demonstrate that the TMEM119-

89 tdTomato signal is detected in the brain and spinal cord, is specific to microglia and is not 
90 detected in peripheral myeloid cells. Using two-photon microscopy, we also show that our

91 Tmem119-tdTomato knock-in mouse strain is reliable for live imaging of microglia in naïve

92 mice as well as in mice that were exposed to brain injury.

93 


\section{2. Materials and Methods}

\section{2.1. Mice}

Tmem119-tdTomato reporter mice were generated in BioCytogen Co, Ltd (Beijing, China).

97 Wild-type C57BL/6J mice were purchased from the Jackson Laboratory (Maine, USA) and

98 were crossed with the Tmem119-tdTomato mice. Animals were housed in the pathogen-free

99 animal facility at Columbia University Medical Center, in accordance with the guidelines of

100 the Committee of Animal Research at Columbia University and the National Institutes of

101 Health animal research guidelines as set forth in the Guide for the Care and Use of

102 Laboratory Animals. All studies were performed in compliance with procedures approved by

103 Columbia University Institutional Animal Care and Use Committee.

\section{2.2. Generation of the Tmem119-tdTomato knock-in mice using CRISPR/Cas9}

105 The Tmem119-tdTomato knock-in mouse strain was generated using CRISPR/Cas9

106 technology. Briefly, eight single-guide RNAs (sgRNA, see supplementary File 1) were

107 designed to target the sequence of the stop codon of murine Tmem119 locus. The activity of

108 the CRISPR/Cas9 was assessed using a UCA kit. The results of genotyping with the UCA kit

109 showed that one sgRNA (sgRNA \#4) was transcribed successfully with required

110 concentration (supplementary File 1). Following embryo microinjection using a

111 micromanipulator, embryos were cultured to the two-cell stage, followed by transfer into

112 pseudopregnant C57BL/6 females. The founders were genetically examined by amplifying

113 sequences spanning the 5' and 3' junction and including the entire inserted transgene. The

114 detailed workflow of the mouse line design, plasmid construction, Southern blot study

115 design, microinjection and founder genotyping is shown (Supplementary File 1).

\section{2.3. DNA isolation and genotyping}

117 Mouse genomic DNA was isolated from tail biopsies $(0.5-1 \mathrm{~cm})$ and following overnight 118 digestion at $55 \square$ into $500 \mathrm{uL}$ of buffer containing $100 \mathrm{mM}$ Tris- $\mathrm{HCl}(\mathrm{pH}$ 8.0), $5 \mathrm{mM}$ EDTA 
119 (pH 8.0), $200 \mathrm{mM} \mathrm{NaCl}, 0.2 \%$ SDS and $0.1 \mathrm{mg} / \mathrm{mL}$ Proteinase K. The samples were gently

120 mixed and cooled down at room temperature for 10-15 min. After centrifugation at 12000

$121 \mathrm{rpm}$ for $15 \mathrm{~min}, 400 \mathrm{uL}$ of the supernatants were collected and gently mixed with the same

122 volume of isopropanol. After centrifugation at $12000 \mathrm{rpm}$ for $10 \mathrm{~min}$, the pellets were

123 washed by gently mixing with $700 \mathrm{uL}$ of $75 \%$ ice-cold ethanol. After centrifugation at 12000

$124 \mathrm{rpm}$ for $5 \mathrm{~min}$, the DNA pellets were harvested and air-dried at room temperature for $5 \mathrm{~min}$.

125 The DNA was dissolved in $100 \mathrm{uL}$ of distilled water at $55 \square$ for two hours, and $100 \mathrm{ng}$ DNA

126 were used for PCR reaction. Wild-type primers (562bp): Forward: 5'-

127 CAGAACCTCCGGTCTCCAGCTAGAG-3'; Reverse: 5'-

128 AGAGAAGTGGTGCGTTAGGGTGAAG-3'; Knock-in primers (299bp): Forward: 5'-

129 CCACCACCTGTTCCTGTACG-3'; Reverse: 5'-

130 AGAGAAGTGGTGCGTtAGGGTGAAG-3'. The PCR conditions were as follows: 1) 95 $\square$

131 for $15 \mathrm{~min}$; 2) 35 cycles at $94 \square$ for $45 \mathrm{sec}, 60 \square$ for $1 \mathrm{~min}$, and $72 \square$ for $1 \mathrm{~min}$; 3) $72 \square$ for 5

132 min. PCR products were separated on 2\% Agarose gel. Detailed analysis of the genotyping

133 strategy is provided (Supplementary File 2).

\section{2.4. RNA isolation and real-time quantitative PCR}

135 Animals were humanely killed by $\mathrm{CO} 2$ and transcardially perfused with ice-cold PBS. The

136 left-side half brains were harvested and frozen in dry ice and stored at -80C. RNA was

137 isolated using RNeasy Plus Micro Kit (\#74034; QIAGEN, USA), and $10 \mathrm{uL}$ of RNA was

138 reversely transcribed into cDNA using the High-Capacity cDNA Reverse Transcription Kit

139 (\#4368814; Thermo Fisher Scientific, USA) following the manufacturer's instructions. The

140 Tmem119 gene expression was detected by real-time quantitative PCR using TaqMan Fast

141 Advanced Master Mix (\#4444557; Thermo Fisher Scientific) and Tmem119 TaqMan Gene

142 Expression Assay (\#Mm00525305_m1; Thermo Fisher Scientific). Gapdh TaqMan Gene

143 Expression Assay (\#Mm99999915_g1; Thermo Fisher Scientific) was applied as an internal 
144 amplification control. The PCR conditions were as follows: $50 \square$ for $2 \mathrm{~min}, 95 \square$ for $2 \mathrm{sec}$,

145 and followed by 40 cycles at $95 \square$ for $1 \mathrm{sec}$ and $60 \square$ for $20 \mathrm{sec}$. The gene expression of

146 Tmem119 relative to Gadph was quantified by the $\Delta \Delta \mathrm{Ct}$ method.

\section{2.5. Immunofluorescence staining}

148 Animals were humanely killed by $\mathrm{CO} 2$ and and transcardially perfused with ice-cold PBS.

149 The right-side half brains were harvested and fixed in 4\% paraformaldehyde (made in PBS;

$150 \# 15710$, Electron Microscopy Sciences, USA) at $4 \square$ for 6 days. After dehydration in $30 \%$

151 sucrose (made in PBS; \#57-50-1, Affymetrix, USA), 30- $\mu$ m thick free-floating sections were

152 sliced using the Cryostat CM3050S (Leica, Germany). For immunostaining, sections were

153 washed in PBS three times (10 min each), and incubated in blocking buffer (3\% BSA

154 (\#A7906, Sigma, USA) in PBS supplemented with 0.1\% Triton X-100 (\#9002-93-1; Sigma))

155 at room temperature for one hour. Primary antibodies (rabbit anti-TMEM119 (1:500;

156 \#ab209064; Abcam, USA), mouse anti-TMEM119 (1:100; \#853302, BioLegend, USA),

157 rabbit anti-Iba1 (1:500; \#019-19741; Wako, Japan), mouse anti-NeuN (1:500; \#MAB377,

158 Millipore, USA), rabbit anti-GFAP (1:500; \#ZO334; Dako, USA), rabbit anti-Olig2 (1:500;

159 \#AB9610; Millipore, USA) diluted in 1\% BSA (in PBS) were further applied to the sections

160 at $4^{\circ} \mathrm{C}$ overnight. After being washed in PBS four times (10 min each), the sections were

161 incubated in species-matched secondary antibodies conjugated with 488 diluted in $1 \%$ BSA

162 (in PBS) at room temperature for one hour. Sections were washed again in PBS for four times

163 (10 min each), and mounted on slides with mounting media with DAPI (\#NC9524612;

164 Vector Laboratories, USA), and imaged using confocal microscope (LSM700, Zeiss,

165 Germany).

$166 \quad$ 2.6. Flow cytometry

167 Animals were humanely killed by $\mathrm{CO} 2$ and blood was collected followed by perfusion with

168 ice-cold PBS. Brain, spinal cord, lung, heart, liver, spleen, kidney and intestine were 
169 harvested, and homogenized in 2\% FBS (in PBS). The cells were collected by centrifuging at

$170300 \mathrm{~g}, 4 \square$ for $10 \mathrm{~min}$ and were resuspended in MACS buffer (1\% FBS in PBS supplemented

171 with $0.4 \% 0.5 \mathrm{M}$ EDTA). The red blood cells were lysed by incubating with the same volume

172 of ACK lysis buffer (\#A1049201, Gibco, USA). Cells were resuspended after being washed

173 twice in MACS buffer and analyzed by BD Accuri C6 flow cytometer (BD Biosciences,

174 USA). The blood samples were incubated with 10× volume of RBC Lysis Solution

175 (\#1045703, QIAGEN) at room temperature for $10 \mathrm{~min}$. After being washed twice in MACS

176 buffer, the cells were resuspended and analyzed by BD Accuri C6. Microglia were isolated as

177 previously described (Butovsky et al., 2014; Olah et al., 2012; Verheijden et al., 2015).

178 Briefly, mice were humanely killed by CO2 and transcardially perfused with ice-cold PBS.

179 The brain tissues were quickly dissected and single cell suspensions were prepared and

180 separated using a 37\%/70\% Percoll gradient (\#GE17-0891-01, GE Healthcare). Myelin was

181 removed from the top layer and mononuclear cells were isolated from the interface. The cells

182 were incubated with mouse anti-CD45-FITC (1:200; \#103108, BioLegend) and mouse anti-

183 CD11b-APC (1:80; \#101212, BioLegend) for 30 minutes on ice in the dark. Cell suspensions

184 were analyzed using the BD FACS Canto (BD Biosciences), and data were analyzed with

185 FlowJo software (TreeStar, USA).

\section{2.7. Two-photon laser injury inside the cortex}

187 A highly localized injury was achieved by focusing a two-photon laser beam ( $\square 1 \mu \mathrm{m}$ in 188 size) in the superficial layer of the cortex through the open skull window. The wavelength

189 of the two-photon laser was set at $800 \mathrm{~nm}$ and the laser power was $\square 60-80 \mathrm{~mW}$ at the 190 sample. The beam was parked at the desired position for approximately 3-5 seconds to 191 create a small injury site as indicated by a bright autofluorescent sphere $(\square 30 \mu \mathrm{m}$ in 192 diameter) around the focal point of the beam. The injury was confined to the area $\square 25-35$ 
$193 \mu \mathrm{m}$ in diameter around the laser focal point, because microglia within this area lost their

194 tdtomato immediately after laser ablation, whereas those $\square 50 \mu \mathrm{m}$ from the injury site still

195 responded to the ablation. The laser-induced focal ablation is a useful injury model, as the

196 site and degree of injury are easy to control, and the response of microglia toward the

197 injury is highly reproducible.

198 2.8. In vivo imaging of microglia with two-photon microscopy

199 To investigate microglia in vivo in intact brain, tdTomato-labeled microglia were imaged

200 by two-photon microscopy through a small craniotomy. Briefly, 2-3 month-old Tmem119-

201 tdTomato mice were anesthetized intraperitoneally with ketamine $(200 \mathrm{mg} / \mathrm{kg}$ body

202 weight) and xylazine (30 mg/kg body weight) in $0.9 \% \mathrm{NaCl}$ solution. A region ( $\square 1 \mathrm{~mm}$ in

203 diameter) over primary sensory cortex was first thinned with a high-speed drill under a

204 dissecting microscope and then opened either with a needle or forceps. A drop ( $\square 200 \mu \mathrm{l}$ )

205 of artificial mouse cerebrospinal fluid (ACSF) was applied on the exposed region for the

206 duration of the experiment. The skull surrounding the open window was attached to a

207 custom-made steel plate to reduce respiratory-induced movement. The animal was placed

208 under a two-photon microscope (Scientifica Hyperscope, East Sussex, UK) while still

209 under anesthesia and temperature maintained with a heating pad $\left(\sim 37^{\circ} \mathrm{C}\right)$. The two-photon

210 laser was tuned to the excitation wavelength for tdTomato $(1000 \mathrm{~nm})$. All experiments

211 were performed using a 1.1-numerical aperture (NA) $25 \times$ objective lens immersed in

212 ACSF to obtain $62.5 \mathrm{X}$ high-magnification $(166.4 \mu \mathrm{m} \times 166.4 \mu \mathrm{m} ; 512 \times 512$ pixels; 1.5 -

$213 \mu \mathrm{m}$ step) images for neuronal analysis. The maximum imaging depth was $\square 120 \mu \mathrm{m}$ from

214 the pial surface. Images were acquired using low laser power $(<30 \mathrm{~mW}$ at the sample $)$ and

215 a low-pass emission filter $(<700 \mathrm{~nm})$.

\section{2.9. Image processing and quantification}


217 All image processing and quantification were performed with Image J software (NIH). All

218 z-stacks of images were projected along the z-axis to recreate a two-dimensional

219 representation of 3D structures. To quantify the microglia response to laser induced injury,

220 we measured the number of microglia processes entering from outer area Y into inner area

221 as a function of time. The number of red pixels in area $\mathrm{X}$ or $\mathrm{Y}$ were measured at each time

222 point $\left(\mathrm{R}_{\mathrm{x}}(\mathrm{t})\right.$ or $\mathrm{R}_{\mathrm{y}}(\mathrm{t})$, and the microglial response was defined as $\mathrm{R}(\mathrm{t})=\left[\mathrm{R}_{\mathrm{x}}(\mathrm{t})\right.$ -

$\left.223 \mathrm{R}_{\mathrm{x}}(0)\right]\left(\right.$ Bennett et al., 2016)/ $\mathrm{R}_{\mathrm{y}}(0)$. To account for signal intensity differences among

224 different experiments, we thresholded every image so that all processes had the maximum

225 value (255), and all background was set to 0 . We then counted the number of white pixels

226 in area $\mathrm{X}$ over time $(\mathrm{Rx}(\mathrm{t}))$ and compared it with the first picture taken immediately after

227 the ablation $\left(\mathrm{R}_{\mathrm{x}}(0)\right)$. The number of white pixels corresponds to the region covered by

228 processes within the area $\mathrm{X}$, and its increase over time provides a measure of the

229 microglial response. To account for the variability in the number of microglia located in

230 the outer area $\mathrm{Y}$ in different experiments, we calculated the microglial response relative to

231 the number of processes in the outer area $\mathrm{Y}$ immediately after the ablation $\left(\mathrm{R}_{\mathrm{y}}(0)\right)$. The

232 microglial response at any time point $(\mathrm{R}(\mathrm{t}))$ is therefore given by $\mathrm{R}(\mathrm{t})=\left(\mathrm{R}_{\mathrm{x}}(\mathrm{t})\right.$ -

$233 \operatorname{Rx}(0)) / \mathrm{R}_{\mathrm{y}}(0)$.

\section{2.10. Data analysis}

235 All data are presented as mean \pm s.e.m., and were analyzed by GraphPad Prism 8. Unpaired

236 Student $t$-test and one-way ANOVA were used for group comparisons. $P<0.05$ was

237 considered statistically significant. 


\section{3. Results}

\section{3.1. Generation and validation of Tmem119-tdTomato reporter mice}

240 Here, we took advantage of the recent identification of TMEM119 as a specific marker of

241 human and murine microglia (Bennett et al., 2016) to generate a new tool for studying the

242 behavior and function of microglia in health and disease. We describe the generation and the

243 characterization of the Tmem119-tdTomato knock-in mouse strain where endogenous

244 Tmem119 expression is preserved. A CRISPR/Cas9 strategy was used to insert tdTomato,

245 preceded by ribosome-skipping peptide porcine teschovirus-1 polyprotein (P2A), upstream of

246 the stop codon of murine Tmem119. Two founder lines were established for Tmem119-

247 tdTomato that were bred with wild-type mice on the C57BL/6 background to generate an on-

248 target sequence (Figure 1A). The data demonstrating the specific insertion of the transgene

249 into the Tmem119 locus are shown (Supplementary File 1). Confirmation of the mouse

250 genotypes comparing WT mice to heterozygous or homozygous lines are shown (Figure 1B

251 and Supplementary File 2). To validate that the transgene insert does not affect endogenous

252 Tmem119 expression, we measured Tmem119 gene expression by quantitative PCR. As

253 expected, we found no difference in Tmem119 mRNA levels in brain homogenates by

254 comparing Tmem119 $9^{t d T /+}$ mice with wild-type controls $(p=0.9996$; Student's $t$-test; Figure

255 1C). Next, to confirm that tdTomato is co-expressed with TMEM119, we used two 256 commercially available anti-TMEM119 antibodies (anti-intracellular and anti-extracellular 257 domain of TMEM119) to track the TMEM119 expression in coronal brain sections from 10 258 months old Tmem1 $19^{\text {tdTomato/+ }}$ mice. Our data showed that both of the antibodies recognized 259 the tdTomato ${ }^{+}$cells, indicating that tdTomato ${ }^{+}$cells readily express TMEM119. 
262 To examine whether tdTomato is predominately expressed in the CNS of Tmem119-

263 tdTomato mice, we analyzed CNS and peripheral tissues and organs by immunofluorescence

264 and flow cytometry. Thus, we collected brain, spinal cord, blood, heart, kidney, lung,

265 intestine, spleen and liver from 3-month old Tmem119 $9^{\text {tdTomato/+ }}$ mice. Immunofluorescence

266 staining showed that tdTomato positive cells were detected throughout the CNS regions,

267 including the olfactory bulb, the prefrontal cortex, the hippocampus, the thalamus, the

268 midbrain, the cerebellum and the spinal cord (Figure 2A-I). In contrast, no tdTomato

269 expression was observed in peripheral tissues such as the heart, the kidney, the lung, the

270 intestine, the spleen and the liver (Figure 2J-O). To confirm these findings, we harvested cell

271 suspensions from these tissues as well as blood and measured the tdTomato expression by

272 flow cytometry. Based on a gating strategy the excludes doublets and debris (Figure 2P), we

273 detected $15.1 \%$ of events as tdTomato positive in the brains of Tmem $119^{\text {tdTomato/+ }}$ mice, but

274 not in the brains of WT mice (Figure 2Q). Consistent with the histological analysis, there

275 was no tdTomato positive cells detected in peripheral tissues (Figure 2S-X) or blood (Figure

276 2R).

277

278 3.3. TMEM119-tdTomato is constantly expressed in adult and aged CNS

279 We have provided evidence to prove spatial expression of TMEM119-tdTomato that is

280 specific to the CNS. We further asked if TMEM119 expression is maintained in aged brains.

281 To address this question, we analyzed tdTomato expression in coronal brain sections from

282 different ages [Postnatal day (P)90, P300 and P540] of Tmem119 $9^{\text {tdTomato/+ }}$ mice by

283 immunofluorescence staining. Our data show that TMEM119-tdTomato expression is

284 expressed in the brain of both young adult and aged mice (Figure 3A-C).

285

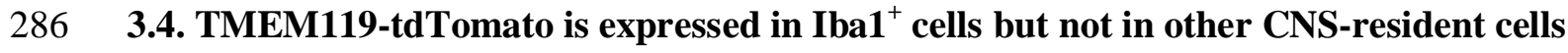


287 Given that tdTomato is specifically expressed in microglia, we sought to examine whether

288 TMEM119 is microglial-specific or is also detectable in other CNS-resident cells such as 289 neurons, astrocytes and oligodendrocytes. To do this, we stained coronal brain sections of

290 Tmem $119^{\text {tdTomato/+ }}$ mice with a marker of each of these three cell types in addition to a 291 microglial marker. Thus, the following antibodies - against NeuN, GFAP, Olig2 and Iba1 292 were tested in the coronal brain sections from of Tmem119 $9^{\text {tdTomato/+ }}$ mice to detect neurons, 293 astrocytes, oligodendrocytes and microglia, respectively. Our data showed that tdTomato 294 expression was only detected in $\mathrm{Iba}^{+}$cells but not in $\mathrm{NeuN}^{+}, \mathrm{GFAP}^{+}$and $\mathrm{Olig}^{+}$cells in the 295 brain (Figure 4A-D). These findings confirm that TMEM119 is exclusively expressed in 296 microglial cells in the brain.

\subsection{TMEM119-tdTomato is specifically expressed in microglia but not in infiltrating} immune cells in adult brain

300 To further confirm that TMEM119-tdTomato ${ }^{+}$cells are indeed microglia, we used a Percoll 301 density gradient technique to isolate microglia from the brain of adult WT and 302 Tmem119 $9^{\text {tdTomato/+ }}$ mice. We then compared the tdTomato ${ }^{+}$cells to the $\mathrm{CD}^{\mathrm{lo}} 5^{\mathrm{lo}} \mathrm{CD} 11 \mathrm{~b}^{+}$ 303 microglia. Based on the gating strategy showed in a representative experiment in Figure 5A304 D, we found $97.8 \%$ of live cells were $\mathrm{CD}_{4} 5^{+}$in WT mice (Figure 5E) relative to $99.4 \%$ in

305 Tmem $119^{\text {tdTomato/+ }}$ mice (Figure 5F). Within the $\mathrm{CD}^{+} 5^{+}$cells, $90.6 \%$ of this population was 306 tdTomato-positive in the Tmem119 $9^{\text {tdTomato/+ }}$ mice (Figure 5H), while no tdTomato was 307 detected in WT mice (Figure 5G). The tdTomato $^{+}$cells from Tmem119 $9^{\text {tdTomato/+ }}$ mice were 308 further analyzed, and we found that they are all $\mathrm{CD} 45^{10} \mathrm{CD} 11 \mathrm{~b}^{+}$microglia (Figure 5I). In the 309 reverse experiment, we found that $97.5 \%$ of $\mathrm{CD} 45^{+}$cells in WT (Figure 5J) and $94.6 \%$ of 310 these cells in Tmem $119^{\text {tdTomato/+ }}$ mice (Figure 5K) are $\mathrm{CD} 45^{10} \mathrm{CD} 11 \mathrm{~b}^{+}$microglia, and $96.6 \%$ 311 of the $\mathrm{CD} 45^{\mathrm{lo}} \mathrm{CD} 11 \mathrm{~b}^{+}$microglia are tdTomato positive in $T$ mem1 $19^{\mathrm{tdTomato} /}$ mice while none 
312 are positive in WT mice (Figure 5L). Overall, our data demonstrate that all tdTomato ${ }^{+}$cells

313 are $\mathrm{CD} 45^{\mathrm{lo}} \mathrm{CD} 11 \mathrm{~b}^{+}$microglia.

315 3.6. Two-photon live imaging of TMEM119-tdTomato ${ }^{+}$microglia in vivo in a laser-

316 induced injury model

317 To validate the Tmem119-tdTomato reporter mice as a useful tool to monitor the activity of

318 microglia in vivo, we elected to use a model of laser-induced injury to monitor microglia

319 response in the intact living brain. Using transcranial two-photon microscopy, we were able

320 to image the behavior of tdTomato-expressing parenchymal microglia through the open skull

321 window of anesthetized Tmem $119^{\text {tdTomato/+ }}$ mice. Thus we performed a small laser ablation,

$322 \sim 30 \mu \mathrm{m}$ in diameter, inside the primary sensory cortex (Figure 6A). Time-lapse images (up

323 to150 minutes) show that microglia formed a spherical containment around the laser lesion

324 site. Within minutes after laser injury, the number of microglia entering from outer area (Y)

325 into inner area $(\mathrm{X})$ is measured as a function of time showing rapid response of microglia to

326 the injury site (Figure 6B, C). During this period, the same cells also retracted those

327 processes that previously lay in directions opposite to the site of injury. Most of the cellular

328 content of each of the immediate neighbors was directed towards the damaged site within the

329 first 1-3 h (Figure 6D, Supplementary Video 1). Length changes of four processes marked

330 in Figure 6D were quantified as function of time (Figure 6E). These results indicate that the

331 Tmem119-tdTomato mouse strain is a viable model to monitor and analyze the activity and

332 the formation of microglia processes in neuroinflammatory disease models. 


\section{4. Discussion}

334 Microglia are becoming the center of attention in the field of neurodegeneration and several

335 clinical trials are currently designed to manipulate microglial function in the context of 336 neurodegenerative diseases (Fu et al., 2019; Mullard, 2018; Subramaniam and Federoff, 337 2017). However, cultured microglia quickly lose their unique microglia signature once out of 338 the CNS, making the ability to study these cells difficult. Here, we present a model which 339 allows us to study microglial behavior in vivo, even in the presence of other innate immune 340 cells. Given the lack of markers that discriminate between microglia and infiltrated myeloid 341 cells that are shown to invade the CNS in neurodegenerative diseases, developing new tools 342 to detect and isolate microglia is becoming of high interest to the research community. In the 343 present study, we report a microglia-specific mouse strain that express the recently identified 344 microglia-specific marker Tmem119 (Bennett et al., 2016; Satoh et al., 2016). A knock-in 345 strategy was used to generate the Tmem119-tdTomato where tdTomato is placed between 346 exon 2 and the 3'UTR of Tmem119 gene thus allowing the detection of TMEM119-positive 347 cells without affecting TMEM119 endogenous expression as shown by comparing Tmem119 348 expression in the brains of Tmem119-tdTomato and wild-type mice. The choice of tdTomato 349 as a reporter protein is because it provides a very bright red fluorescence $(\sim 3$ fold higher than 350 EGFP)(Shaner et al., 2005) that is ideal for live imaging and potentially for generating double 351 reporter mice with GFP mice for studying microglia in relation to other GFP-labeled cell 352 types.

353 Using immunofluorescence and flow cytometry analysis we provide evidence that tdTomato 354 selectively labels microglial cells in the CNS but not peripheral myeloid cells. Satoh et al. 355 showed that TMEM119 could discriminate brain-residing microglial cells from circulating 356 macrophages, as this protein is expressed exclusively on ramified and amoeboid microglial 357 cells in the brain (Satoh et al., 2016). Additionally, we validate the specificity of two 
358 commercially available anti-TMEM119 antibodies available at Abcam and Biolegend that

359 stain the intracellular and extracellular domains of TMEM119, respectively. In adult

360 Tmem119-tdTomato reporter mice, we found that TMEM119 is expressed throughout the

361 brain and the spinal cord. Strikingly, we did not detect the tdTomato signal in peripheral

362 lymphoid organs and the blood. Bennett and colleagues generated monoclonal antibodies to

363 the intracellular and extracellular domains of TMEM119 that enable the immunolabeling of

364 microglia in histological sections as well as isolation of microglia by FACS (Bennett et al.,

365 2016). The study by Bennett et al. showed that Tmem119 immunoreactivity is developmentally

366 regulated as Tmem119 expression did not appear until postnatal day 3 and 6 (Bennett et al.,

367 2016). These findings are in agreement with the present study where we did not detect

368 tdTomato expression in the brain of newborn mice (data not shown).

369 During the past few years and with the availability of instrumental gene profiling techniques

370 that can process very small numbers of cells, mainly from human autopsies and biopsies, led

371 to a series of reports generating transcriptomics profiling of human and murine microglia at

372 the bulk and the single-cell levels (Butovsky et al., 2014; Chiu et al., 2013; Hickman et al.,

373 2013; Marta Olah, 2019; Masuda et al., 2019; Olah et al., 2018; Zhang et al., 2014). Several

374 microglia clusters have been identified with specific gene signatures that are associated with

375 homeostatic and active microglia. In our recent study examining the gene signature of the

376 aged human brain using fresh brain autopsies from aged subjects, we found that Tmem119

377 gene expression is found in microglia purified from aged human brain (Olah et al., 2018).

378 This is consistent with our present data in the Tmem119-tdTomato mice where TMEM119

379 expression is readily present in aged microglia (1.6 years old mice) at the protein level.

380 More recently, given the heterogeneous nature of the human brain, we have utilized a

381 single cell RNA-seq approach to analyze microglia heterogeneity across several

382 neurological disorders including Alzheimer's disease, Parkinson's disease and epilepsy. 
383 Computational analysis identified nine distinct microglia subsets that express different

384 levels of Tmem119 (Marta Olah, 2019). In our Tmem119-tdTomato mice, although

385 TMEM119 expression was detected throughout the healthy adult and aged brains, it

386 remains possible that TMEM119 expression will be regulated in a disease state where

387 microglia are subject to neuroinflammatory stimuli. Another recent study reporting single-

388 cell RNA-seq analyses of microglia in mice and humans showed that Tmem119 is among

389 the most highly differentially regulated genes during development as it is highly expressed

390 in postnatal microglia. The authors found that Tmem119 is expressed in homeostatic

391 microglia clusters in healthy brains whereas microglia clusters that are associated with

392 multiple sclerosis and are characterized as activated clusters express lower levels of

393 Tmem119 (Masuda et al., 2019). Single-cell RNA-seq approaches are an instrumental

394 approach to define heterogeneous cell subsets. Crossing the Tmem119-tdTomato mice

395 with mouse models of neurodegenerative diseases such as Alzheimer's or Parkinson's

396 disease will provide valuable information about the different subsets of TMEM119-

397 positive microglia in a disease state.

398 In addition to the goals discussed above that lead to the generation of microglia-specific

399 reporter mice based on the TMEM119 approach, we were interested in having in hand a

400 reporter model where we can image microglia in vivo in live animals. Our two-photon

401 imaging data demonstrate that the Tmem119-tdTomato mice are an excellent model for live

402 observation of microglia to analyze their response to any type of stimulation or stress as

403 reported using laser-induced injury. Indeed, our two-photon analysis reported the number of

404 tdTomato-positive microglia processes entering the site of injury, processes formation and

405 their retraction vis-à-vis the site of injury. Our findings suggest that the Tmem119-tdTomato

406 mouse strain is an excellent model for imaging and analyzing microglia activity alone or in 
407 relation to other CNS-resident or infiltrated immune cells in disease models such as multiple

408 sclerosis, Parkinson's disease and Alzheimer's disease.

409 In conclusion, our study provides a microglia-specific reporter mouse model based on

410 the Tmem119 microglia marker. This Tmem119-tdTomato mouse strain will be a critical tool

411 for microglia-focused research in health and disease. Additionally, crossing this mouse strain

412 with other reporter mice expressing other fluorescent proteins specific for CNS-resident cells

413 and peripheral immune cells will provide unique mouse models to study the interaction

414 between microglia and other CNS resident or infiltrated cells. 
bioRxiv preprint doi: https://doi.org/10.1101/665893; this version posted June 10, 2019. The copyright holder for this preprint (which was not certified by peer review) is the author/funder. All rights reserved. No reuse allowed without permission.

\section{Conflicts of interest}

421 Samuel Hasson was employed by Pfizer and is a current employee of Amgen.

\section{Acknowledgments}

424 We thank Pfizer for the generous gift of the Tmem119-tdTomato reporter mice. Research 425 reported in this publication was supported by a grant from the Thompson Family Foundation 426 (W.E.).

427 
Arcuri, C., Mecca, C., Bianchi, R., Giambanco, I., Donato, R., 2017. The Pathophysiological Role of Microglia in Dynamic Surveillance, Phagocytosis and Structural Remodeling of the Developing CNS. Front Mol Neurosci 10, 191.

Bachiller, S., Jimenez-Ferrer, I., Paulus, A., Yang, Y., Swanberg, M., Deierborg, T., BozaSerrano, A., 2018. Microglia in Neurological Diseases: A Road Map to Brain-Disease Dependent-Inflammatory Response. Front Cell Neurosci 12, 488. Bennett, M.L., Bennett, F.C., Liddelow, S.A., Ajami, B., Zamanian, J.L., Fernhoff, N.B., Mulinyawe, S.B., Bohlen, C.J., Adil, A., Tucker, A., Weissman, I.L., Chang, E.F., Li, G., Grant, G.A., Hayden Gephart, M.G., Barres, B.A., 2016. New tools for studying microglia in the mouse and human CNS. Proc Natl Acad Sci U S A 113, E1738-1746.

Boche, D., Perry, V.H., Nicoll, J.A., 2013. Review: activation patterns of microglia and their identification in the human brain. Neuropathol Appl Neurobiol 39, 3-18.

Butovsky, O., Jedrychowski, M.P., Moore, C.S., Cialic, R., Lanser, A.J., Gabriely, G., Koeglsperger, T., Dake, B., Wu, P.M., Doykan, C.E., Fanek, Z., Liu, L., Chen, Z., Rothstein, J.D., Ransohoff, R.M., Gygi, S.P., Antel, J.P., Weiner, H.L., 2014. Identification of a unique TGF-beta-dependent molecular and functional signature in microglia. Nat Neurosci 17, 131143.

Chiu, I.M., Morimoto, E.T., Goodarzi, H., Liao, J.T., O'Keeffe, S., Phatnani, H.P., Muratet, M., Carroll, M.C., Levy, S., Tavazoie, S., Myers, R.M., Maniatis, T., 2013. A neurodegeneration-specific gene-expression signature of acutely isolated microglia from an amyotrophic lateral sclerosis mouse model. Cell Rep 4, 385-401.

Colonna, M., Butovsky, O., 2017. Microglia Function in the Central Nervous System During Health and Neurodegeneration. Annu Rev Immunol 35, 441-468.

452 Fahrenhold, M., Rakic, S., Classey, J., Brayne, C., Ince, P.G., Nicoll, J.A.R., Boche, D., Mrc, 453 C., 2018. TREM2 expression in the human brain: a marker of monocyte recruitment? Brain 454 Pathol 28, 595-602.

455 Fu, W.Y., Wang, X., Ip, N.Y., 2019. Targeting Neuroinflammation as a Therapeutic Strategy 456 for Alzheimer's Disease: Mechanisms, Drug Candidates, and New Opportunities. ACS Chem 457 Neurosci 10, 872-879.

458 Gosselin, D., Skola, D., Coufal, N.G., Holtman, I.R., Schlachetzki, J.C.M., Sajti, E., Jaeger, 459 B.N., O'Connor, C., Fitzpatrick, C., Pasillas, M.P., Pena, M., Adair, A., Gonda, D.D., Levy, 460 M.L., Ransohoff, R.M., Gage, F.H., Glass, C.K., 2017. An environment-dependent transcriptional network specifies human microglia identity. Science 356. Graeber, M.B., Streit, W.J., 1990. Microglia: immune network in the CNS. Brain Pathol 1, 25.

Hanisch, U.K., Kettenmann, H., 2007. Microglia: active sensor and versatile effector cells in the normal and pathologic brain. Nat Neurosci 10, 1387-1394.

Hickman, S.E., Kingery, N.D., Ohsumi, T.K., Borowsky, M.L., Wang, L.C., Means, T.K., El Khoury, J., 2013. The microglial sensome revealed by direct RNA sequencing. Nat Neurosci 16, 1896-1905.

Hisa, I., Inoue, Y., Hendy, G.N., Canaff, L., Kitazawa, R., Kitazawa, S., Komori, T., Sugimoto, T., Seino, S., Kaji, H., 2011. Parathyroid hormone-responsive Smad3-related factor, Tmem119, promotes osteoblast differentiation and interacts with the bone morphogenetic protein-Runx2 pathway. J Biol Chem 286, 9787-9796.

Katsumoto, A., Lu, H., Miranda, A.S., Ransohoff, R.M., 2014. Ontogeny and functions of central nervous system macrophages. J Immunol 193, 2615-2621.

Konishi, H., Kobayashi, M., Kunisawa, T., Imai, K., Sayo, A., Malissen, B., Crocker, P.R., Sato, K., Kiyama, H., 2017. Siglec-H is a microglia-specific marker that discriminates 
477 microglia from CNS-associated macrophages and CNS-infiltrating monocytes. Glia 65, 1927-

4781943.

479 Lawson, L.J., Matyszak, M.K., Perry, V.H., 1993. Lessons from microglia in special sites.

$480 \quad$ Clin Neuropathol 12, 310-313.

481 Marta Olah, V.M., Naomi Habib, Mariko Taga, Christina Yung, Maria Cimpean, Anthony

482 Khairalla, Danielle Dionne, Sarah Hopp, Matthew P. Frosch, Bradley T. Hyman, Thomas

483 Beach, Rani Sarkis, Garth R Cosgrove, Jeffrey Helgager, Jeffrey A. Golden, Page B. Pennell,

484 Julie A. Schneider, David A. Bennett, Aviv Regev, Wassim Elyaman, Elizabeth M.

485 Bradshaw, Philip L. De Jager, 2019. A single cell-based atlas of human microglial states

486

487

488

489

490

491

492

493

494

495

496

497

498 reveals associations with neurological disorders and histopathological features of the aging brain. BioRxiv.org.

Masuda, T., Sankowski, R., Staszewski, O., Bottcher, C., Amann, L., Sagar, Scheiwe, C., Nessler, S., Kunz, P., van Loo, G., Coenen, V.A., Reinacher, P.C., Michel, A., Sure, U., Gold, R., Grun, D., Priller, J., Stadelmann, C., Prinz, M., 2019. Spatial and temporal heterogeneity of mouse and human microglia at single-cell resolution. Nature 566, 388-392. Meyer-Luehmann, M., Prinz, M., 2015. Myeloid cells in Alzheimer's disease: culprits, victims or innocent bystanders? Trends Neurosci 38, 659-668.

Mullard, A., 2018. Microglia-targeted candidates push the Alzheimer drug envelope. Nat Rev Drug Discov 17, 303-305.

Olah, M., Amor, S., Brouwer, N., Vinet, J., Eggen, B., Biber, K., Boddeke, H.W., 2012. Identification of a microglia phenotype supportive of remyelination. Glia 60, 306-321. Olah, M., Patrick, E., Villani, A.C., Xu, J., White, C.C., Ryan, K.J., Piehowski, P., Kapasi, A., Nejad, P., Cimpean, M., Connor, S., Yung, C.J., Frangieh, M., McHenry, A., Elyaman, W., Petyuk, V., Schneider, J.A., Bennett, D.A., De Jager, P.L., Bradshaw, E.M., 2018. A transcriptomic atlas of aged human microglia. Nat Commun 9, 539.

502 Pena-Altamira, E., Prati, F., Massenzio, F., Virgili, M., Contestabile, A., Bolognesi, M.L.,

503 Monti, B., 2016. Changing paradigm to target microglia in neurodegenerative diseases: from

504 anti-inflammatory strategy to active immunomodulation. Expert Opin Ther Targets 20, 627-

505640.

506 Pluvinage, J.V., Haney, M.S., Smith, B.A.H., Sun, J., Iram, T., Bonanno, L., Li, L., Lee, 507 D.P., Morgens, D.W., Yang, A.C., Shuken, S.R., Gate, D., Scott, M., Khatri, P., Luo, J.,

508 Bertozzi, C.R., Bassik, M.C., Wyss-Coray, T., 2019. CD22 blockade restores homeostatic

509 microglial phagocytosis in ageing brains. Nature 568, 187-192.

510 Satoh, J., Kino, Y., Asahina, N., Takitani, M., Miyoshi, J., Ishida, T., Saito, Y., 2016.

511 TMEM119 marks a subset of microglia in the human brain. Neuropathology 36, 39-49.

512 Shaner, N.C., Steinbach, P.A., Tsien, R.Y., 2005. A guide to choosing fluorescent proteins.

513 Nat Methods 2, 905-909.

514 Subramaniam, S.R., Federoff, H.J., 2017. Targeting Microglial Activation States as a

515 Therapeutic Avenue in Parkinson's Disease. Front Aging Neurosci 9, 176.

516 Verheijden, S., Beckers, L., Casazza, A., Butovsky, O., Mazzone, M., Baes, M., 2015.

517 Identification of a chronic non-neurodegenerative microglia activation state in a mouse model

518 of peroxisomal $\beta$-oxidation deficiency. Glia 63, 1606-1620.

519 Wang, S., Colonna, M., 2019. Microglia in Alzheimer's disease: A target for immunotherapy.

520 J Leukoc Biol.

521 Wu, Y., Dissing-Olesen, L., MacVicar, B.A., Stevens, B., 2015. Microglia: Dynamic

522 Mediators of Synapse Development and Plasticity. Trends Immunol 36, 605-613.

523 Zhang, Y., Chen, K., Sloan, S.A., Bennett, M.L., Scholze, A.R., O'Keeffe, S., Phatnani, H.P., 524 Guarnieri, P., Caneda, C., Ruderisch, N., Deng, S., Liddelow, S.A., Zhang, C., Daneman, R., 525 Maniatis, T., Barres, B.A., Wu, J.Q., 2014. An RNA-sequencing transcriptome and splicing 
bioRxiv preprint doi: https://doi.org/10.1101/665893; this version posted June 10, 2019. The copyright holder for this preprint (which was not certified by peer review) is the author/funder. All rights reserved. No reuse allowed without permission.

526 database of glia, neurons, and vascular cells of the cerebral cortex. J Neurosci 34, 11929-

52711947.

528 


\section{Figure Legends}

530 Figure 1. Generation and validation of Tmem119-tdTomato reporter mice. (A) Schematic

531 of engineering strategy to create the Tmem119-tdTomato reporter mice. A DNA sequence of 532 GSG-3xFlag-P2A-tdTomato was inserted between the exon 2 and 3'UTR in the Tmem119 533 gene by CRISPR/Cas9 technology. E, exon; UTR, untranslated region. (B) A representative 534 genotyping result showing the genotype of WT (with a 562-bp size band), Tmem119 $9^{\text {tdTomato/+ }}$

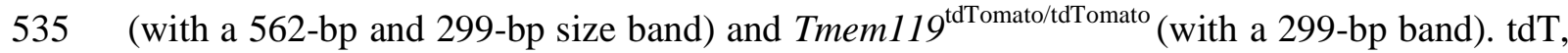
536 tdTomato. (C) The gene expression of Tmem119 relative to Gapdh in central nervous system 537 was detected between 3-month old WT and Tmem119 $9^{\text {tdTomato/+ }}$ mice $(n=3$ each). Data are 538 presented in mean \pm s.e.m. (D) TMEM119 (green) was stained in coronal brain sections from 539 10-month old Tmem119 $9^{\text {tdTomato/+ }}$ mice by an intracellular (left; clone 28-3, Abcam) and an 540 extracellular (right; clone A16075D, BioLegend) antibodies. Red, tdTomato. Yellow arrows, 541 colocalized staining. White arrows, uncolocalized staining. Scale bar, $50 \mu \mathrm{m}$.

543 Figure 2. TdTomato is expressed in the adult CNS but not in the blood nor in

544 peripheral tissues. A representative image of sagittal section in brain (A) and coronal 545 section in spinal cord $(\mathrm{H})$ from 3-month old Tmem119 $9^{\text {tdTomato/+ }}$ mice. Sections: $16-\mu \mathrm{m}$ thick. 546 Scale bar, $500 \mu \mathrm{m}$. TdTomato (red) were detected in cells through over different regions of 547 central nervous system, such as olfactory bulb (B), prefrontal cortex (C), hippocampus (D), 548 thalamus $(\mathbf{E})$, midbrain $(\mathbf{F})$, cerebellum $(\mathbf{G})$ and spinal cord $(\mathbf{I})$. Sections were mounted in 549 media with DAPI (blue). Scale bar, $50 \mu \mathrm{m}$. TdTomato were not detected in cells in peripheral 550 tissues such as heart $(\mathbf{J})$, kidney $(\mathbf{K})$, lung $(\mathbf{L})$, intestine $(\mathbf{M})$, spleen $(\mathbf{N})$ and liver $(\mathbf{O})$.

551 Sections, 16- $\mu \mathrm{m}$ thick. Scale bar, $50 \mu \mathrm{m}$. TdTomato were measured in CNS, blood and 552 peripheral tissues from 3-month old Tmem1 $19^{\text {tdTomato/+ }}$ mice by flow cytometry, and tdTomato 553 positive cells were only detected in CNS tissues $(\mathbf{Q})$ but not in blood $(\mathbf{R})$ and peripheral 
554 tissues such as heart $(\mathbf{S})$, kidney $(\mathbf{T})$, lung $(\mathbf{U})$, intestine $(\mathbf{V})$, spleen $(\mathbf{W})$ and liver $(\mathbf{X})$, based

555 on a gating strategy (P). SSC-A, side scatter (area); FSC-A, forward scatter (area); SSC-H,

556 side scatter (hight); FSC-H, forward scatter (hight); CNS, central nervous system; WT, wild 557 type; tdT, tdTomato.

559 Figure 3. TdTomato is consistently expressed in the adult and aged CNS. TdTomato ${ }^{+}$

560 cells (red) were detectable in the coronal brain sections from postnatal 90 days (A), 300 days

561 (B) and 540 days (C). a, b, and c, are amplified images from each staining. P, postnatal; VI,

562 the fourth layer of neocortex; CA1, the first region of hippocampal circuit. Sections, 30- $\mu \mathrm{m}$

563 thick. Scale bar, $100 \mu \mathrm{m}$.

Figure 4. TdTomato is specifically expressed in $\mathrm{Iba1}^{+}$microglia/macrophages but not in other CNS-resident cells. TdTomato $^{+}$cells (red) were colocalized with

567 microglia/macrophage marker $\mathrm{Iba1}^{+}$cells (A, green), but not neuronal marker $\mathrm{NeuN}^{+}(\mathbf{B}$, 568 green), astrocyte marker $\mathrm{GFAP}^{+}\left(\mathbf{C}\right.$, green) or oligodendrocyte marker Olig2 ${ }^{+}(\mathbf{D}$, green $)$ cells

569 in the coronal brain sections of adult Tmem $119^{\text {tdTomato/+ }}$ mice by immunofluorescence staining.

570 ( $n=3$ sections per mouse, $N=3$ mice). Red, tdTomato. Blue, DAPI. a, b, c, and d, are 571 amplified images from each staining. VI, the fourth layer of neocortex; CA1, the first region 572 of hippocampal circuit. Arrowheads, colocalized staining. Sections, 30- $\mu$ m thick. Scale bar, $573100 \mu \mathrm{m}$.

575 Figure 5. TdTomato is specifically expressed in microglia but not in infiltrating immune

576 cells in the adult brain. (A-D) Flow cytometry analysis to gate out cells of interest (A), 577 single cells $(\mathbf{B}, \mathbf{C})$ and live cells $(\mathbf{D})$. (E-I) tdTomato $^{+}$cells in the brain tissues of WT $(\mathbf{G})$ vs 578 Tmem119 $9^{\text {tdTomato/+ }}(\mathbf{H})$ mice are gated based on the $\mathrm{CD} 45^{+}$cells in WT $(\mathbf{E})$ or 
579 Tmem1 19 $9^{\text {tdTomato/+ }}(\mathbf{F})$ mice. $\mathrm{CD} 45^{\text {lo }} \mathrm{CD} 11 \mathrm{~b}^{+}$cells $(\mathbf{I})$ were further gated out of tdTomato ${ }^{+}$cells

580 (H) in Tmem119 $9^{\text {tdTomato/+ }}$ mice. $(\mathbf{E}, \mathbf{F}, \mathbf{J}-\mathbf{L}) \mathrm{CD}^{\mathrm{T}} 5^{\mathrm{lo}} \mathrm{CD} 11 \mathrm{~b}^{+}$cells $(\mathbf{J}, \mathbf{K})$ were gated out of

$581 \mathrm{CD} 45^{+}$cells in WT $(\mathbf{E})$ or Tmem119 $9^{\text {tdTomato/+ }}(\mathbf{F})$ mice, and the tdTomato ${ }^{+}$cells in WT vs

582 Tmem $119^{\text {tdTomato/+ }}$ mice $(\mathbf{L})$ were further gated based on the $\mathrm{CD} 45^{\text {lo }} \mathrm{CD} 11 \mathrm{~b}^{+}$cells $(\mathbf{J}, \mathbf{K})$. SSC-

583 A, side scatter (area); SSC-H, side scatter (hight); SSC-W, side scatter (width); FSC-A,

584 forward scatter (area); FSC-H, forward scatter (hight); FSC-W, forward scatter (width); WT,

585 wild type; tdT, tdTomato; $\mathrm{CD} 45^{\text {lo }}$, low expression of CD45. The number beside or within the

586 gate indicates percentage of cells in each plot.

Figure 6. Two-photon live imaging of tdTomato $^{+}$microglia in vivo after laser-mediated

589 injury. (A) After a localized ablation inside the primary sensory cortex, neighboring

590 Tmem119-tdTonmato ${ }^{+}$microglia respond quickly with extended processes and bulbous

591 termini. Time-lapse images show that microglia formed a spherical containment around the

592 laser lesion site. Scale bar, $30 \mu \mathrm{m}$. (B) To quantify the laser injury-induced microglial

593 response, the number of microglia processes entering from outer area $\mathrm{Y}$ into inner area $\mathrm{X}$ is

594 measured as a function of time. The number of red pixels in area X or Y were measured at

595 each time point $\left(R_{x}(t)\right.$ or $R_{y}(t)$, and the microglial response was defined as $R(t)=\left[R_{x}(t)\right.$ -

$\left.596 \mathrm{R}_{\mathrm{x}}(0)\right] / \mathrm{R}_{\mathrm{y}}(0)$. Scale bar, $30 \mu \mathrm{m}$. (C) Microglia response over 1 hour after laser injury. (D) The

597 same cell extends its processes towards the laser lesion site (marked by yellow $\mathrm{x}$ ), and

598 retracts the ones in the opposite direction to the injured site. Scale bar, $10 \mu \mathrm{m}$. (E) Length

599 changes of four processes marked in $\mathbf{D}$ as a function of time. Scale bar, $10 \mu \mathrm{m}$.

600

601 Supplementary data

602 Supplementary file 1: Detailed workflow for the design and the generation of the

603 Tmem119-tdTomato knock-in mice. Five sections are provided to highlight the workflow of 
604 the mouse generation starting from the targeting strategy using CRISPR/Cas9 technology, the

605 Cas9/sgRNA plasmid contruction and UCA assay, targeting vector construction \& Southern

606 blot strategy design, sgRNA preparation, the zygote microinjection \& founder genotyping.

607

608 Supplementary file 2: Genotyping guide of the Tmem119-tdTomato knock-in mice. This

609 report includes primer design strategy, primer sequences and predicted product sizes, PCR

610 reaction system \& conditions, gel images, and determination of genotype from the gel image

611 results.

612

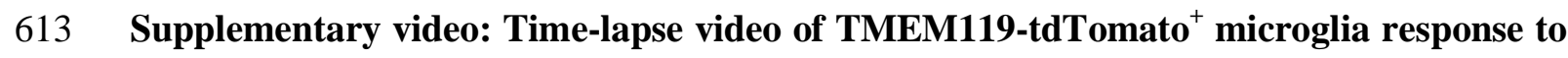

614 laser-induced injury. After a localized ablation inside the primary sensory cortex,

615 neighboring tdTomato ${ }^{+}$microglia respond quickly with extended processes and bulbous

616 termini.

617

618

619 


\section{Figure 1}

A

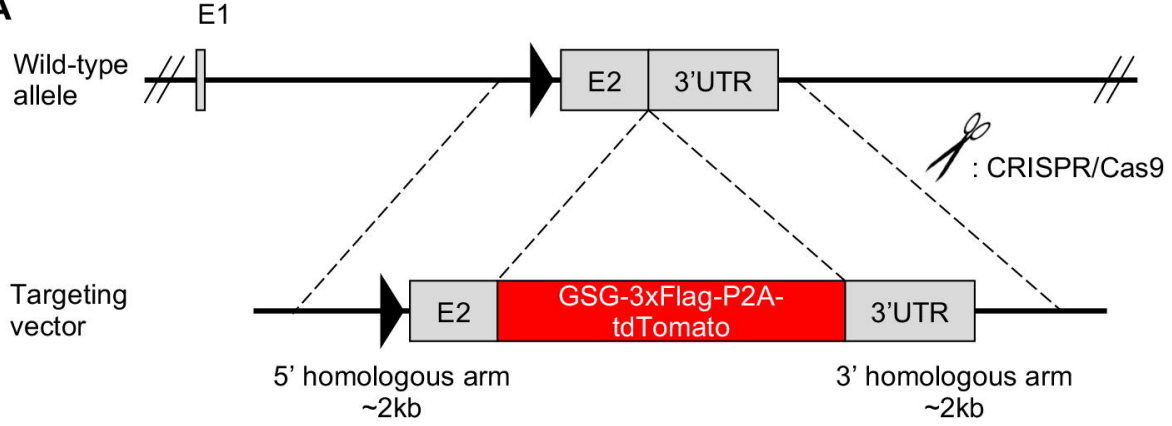

E1

Targeted

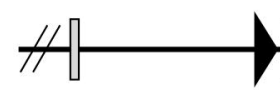

\section{E2}

GSG-3xFlag-P2A-

3'UTR

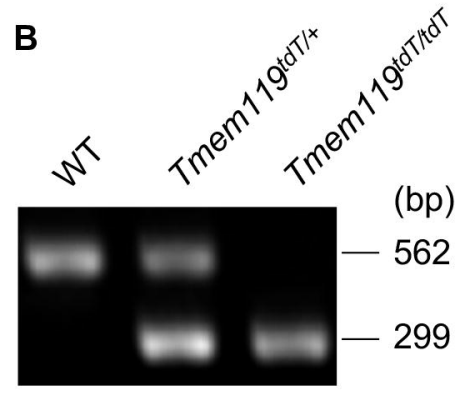

D

Intracellular antibody

Extracellular antibody

C

WT
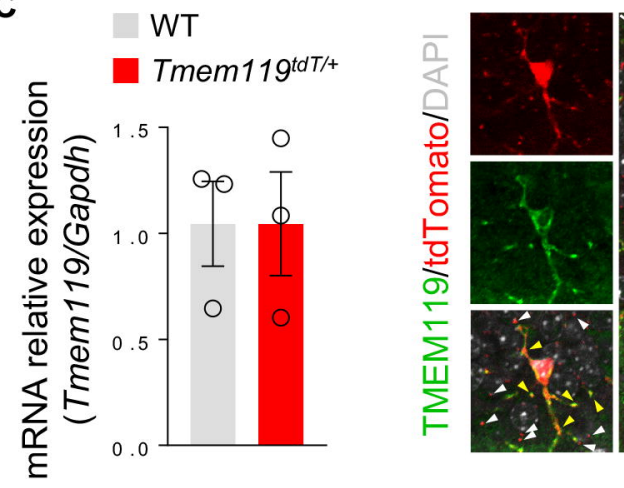

(clone 28-3; Abcam) (clone A16075D; BioLegend)
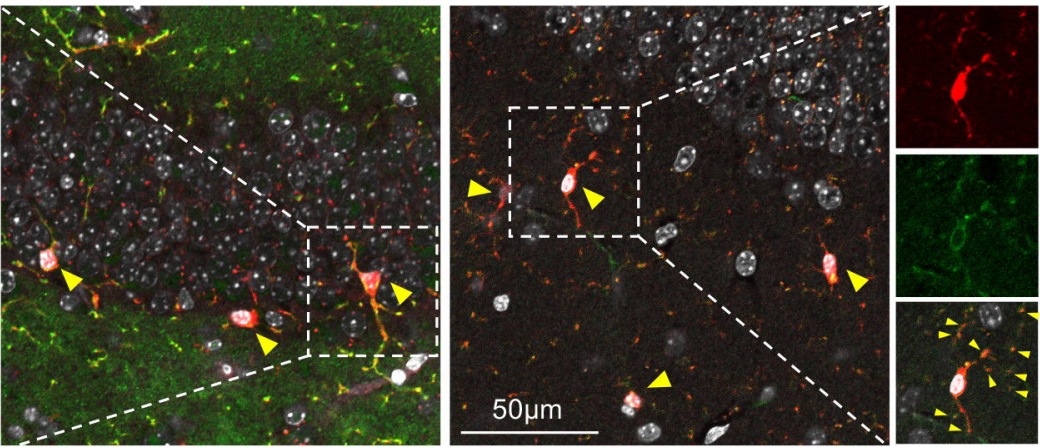
Figure 2
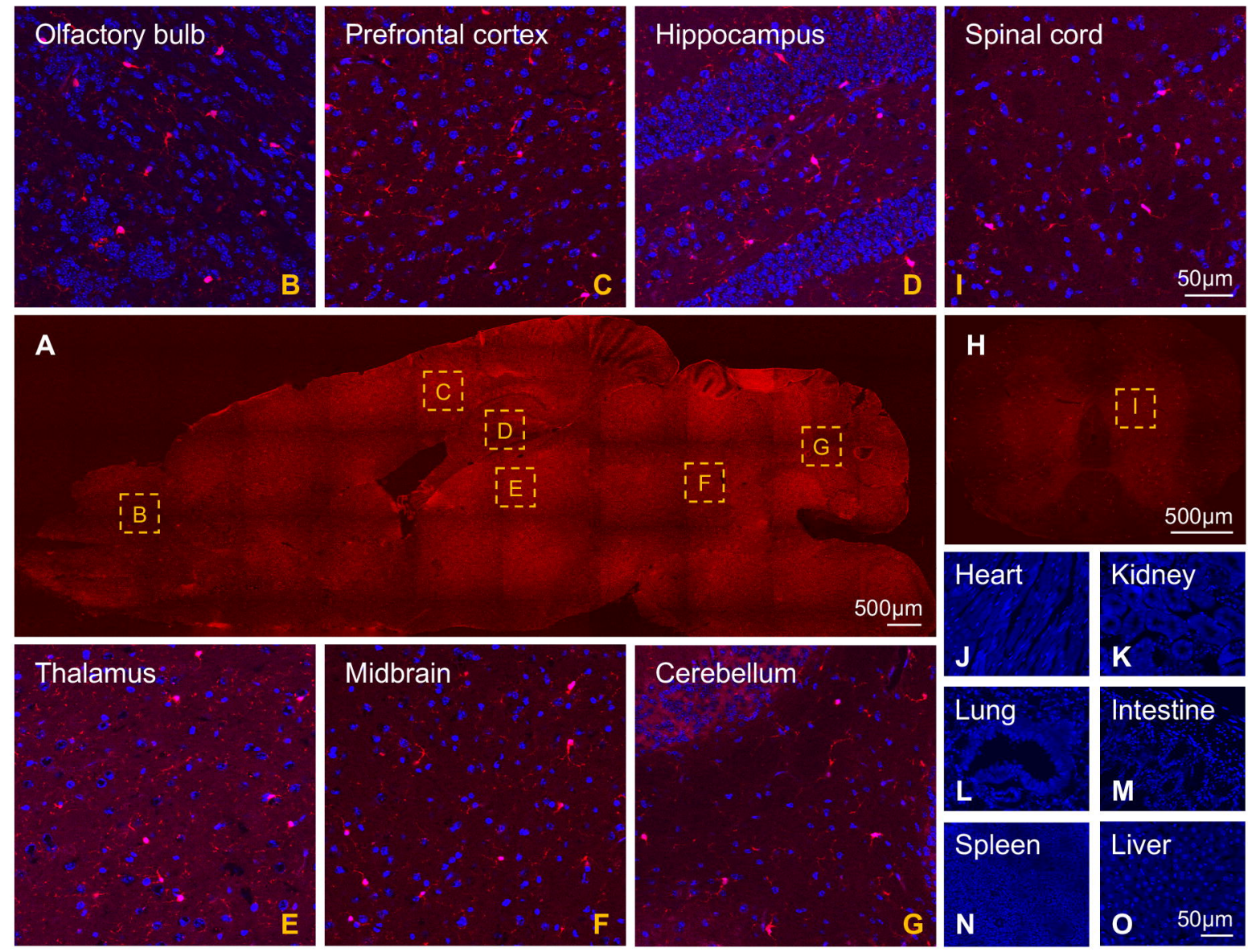

H
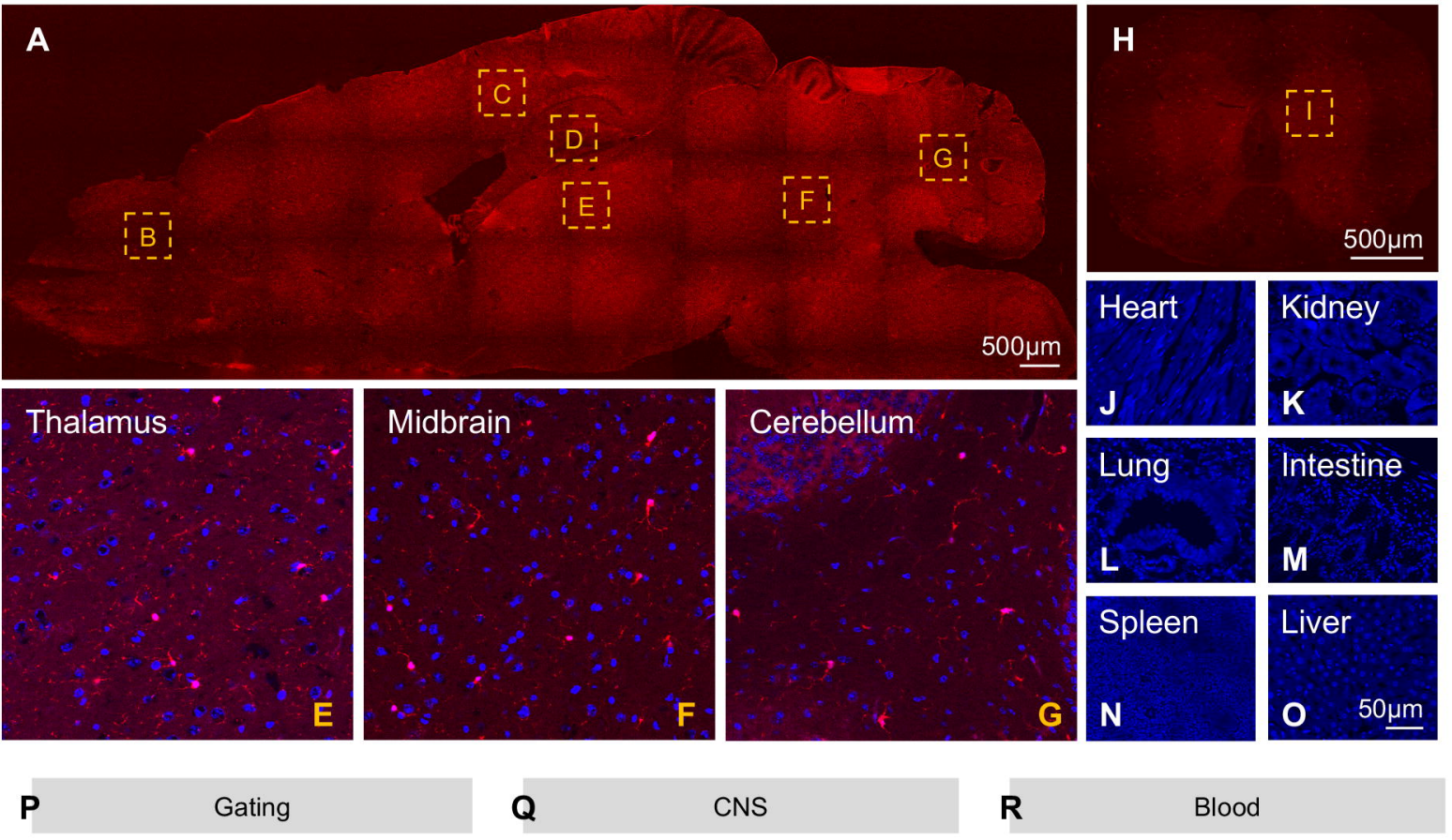

$500 \mu \mathrm{m}$
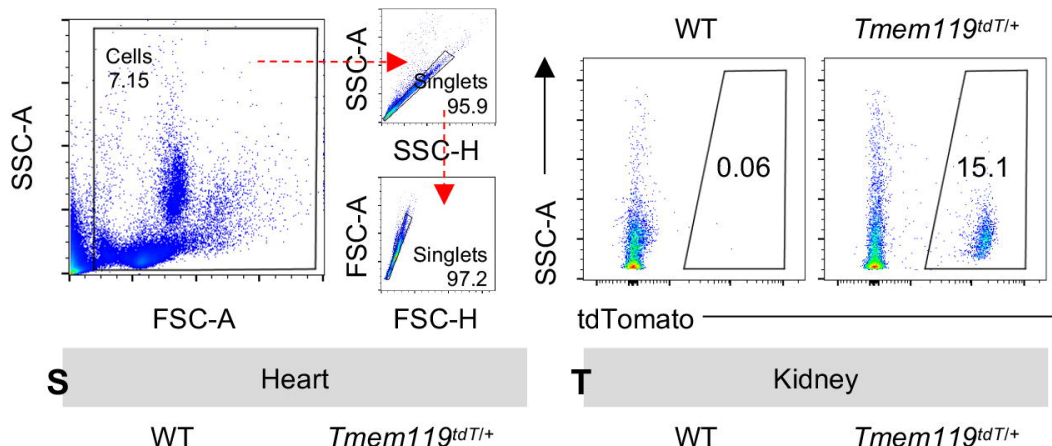

R Blood
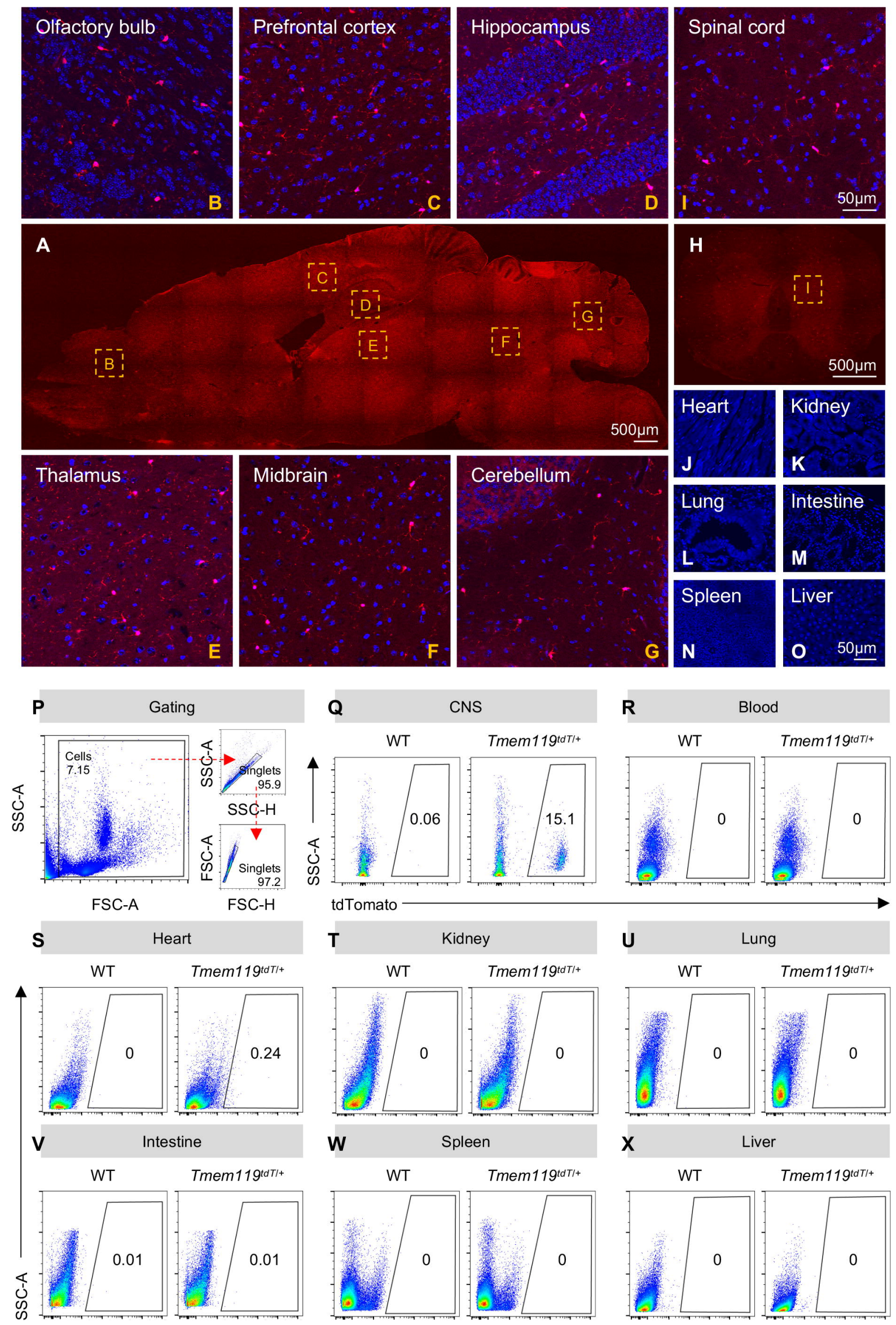


\section{Figure 3}

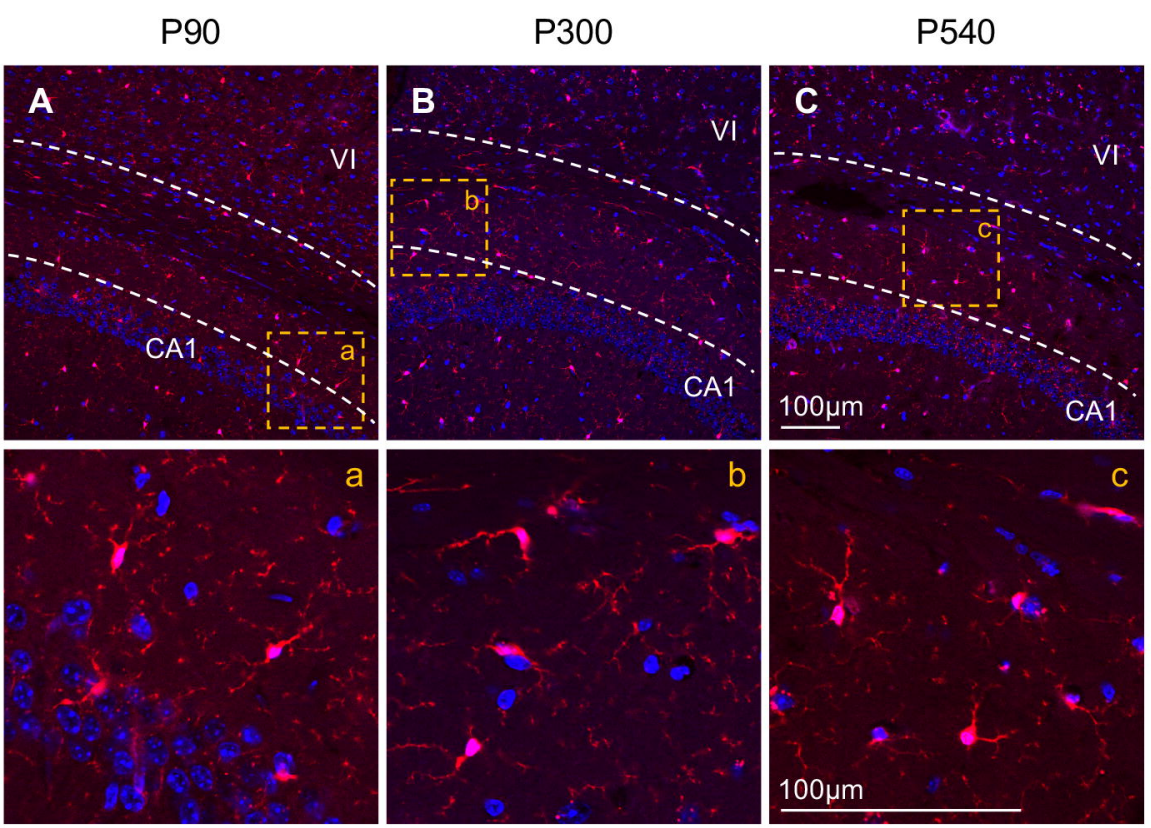


Figure 4

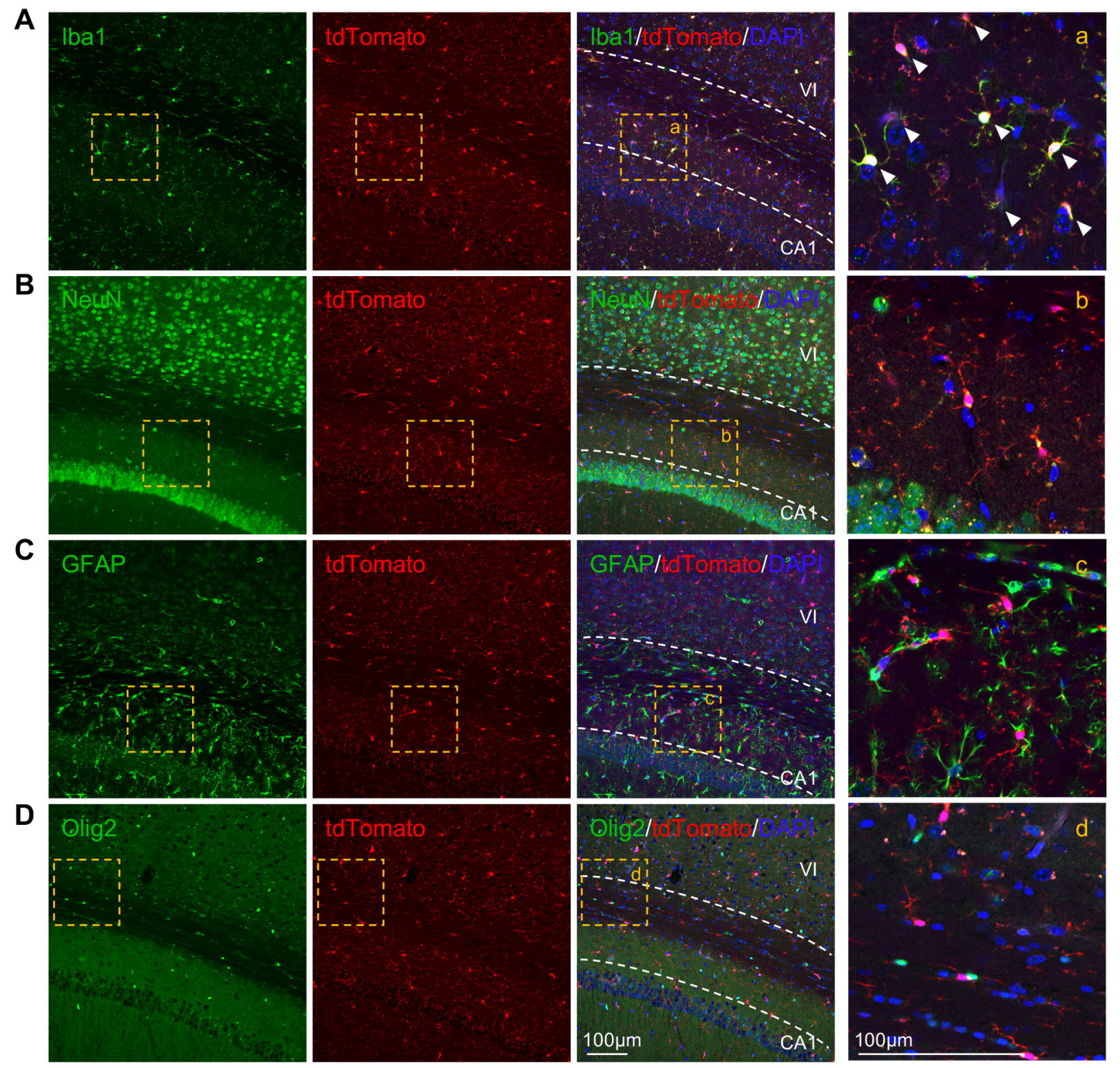


Figure 5

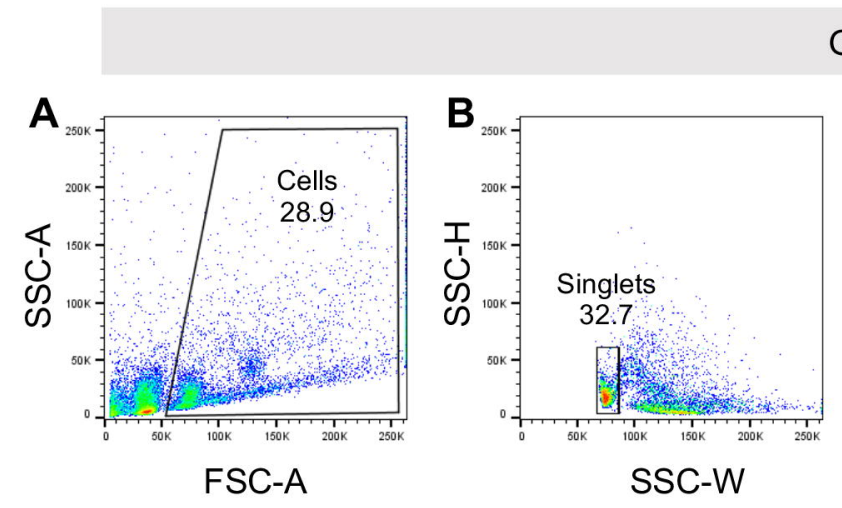

Gating

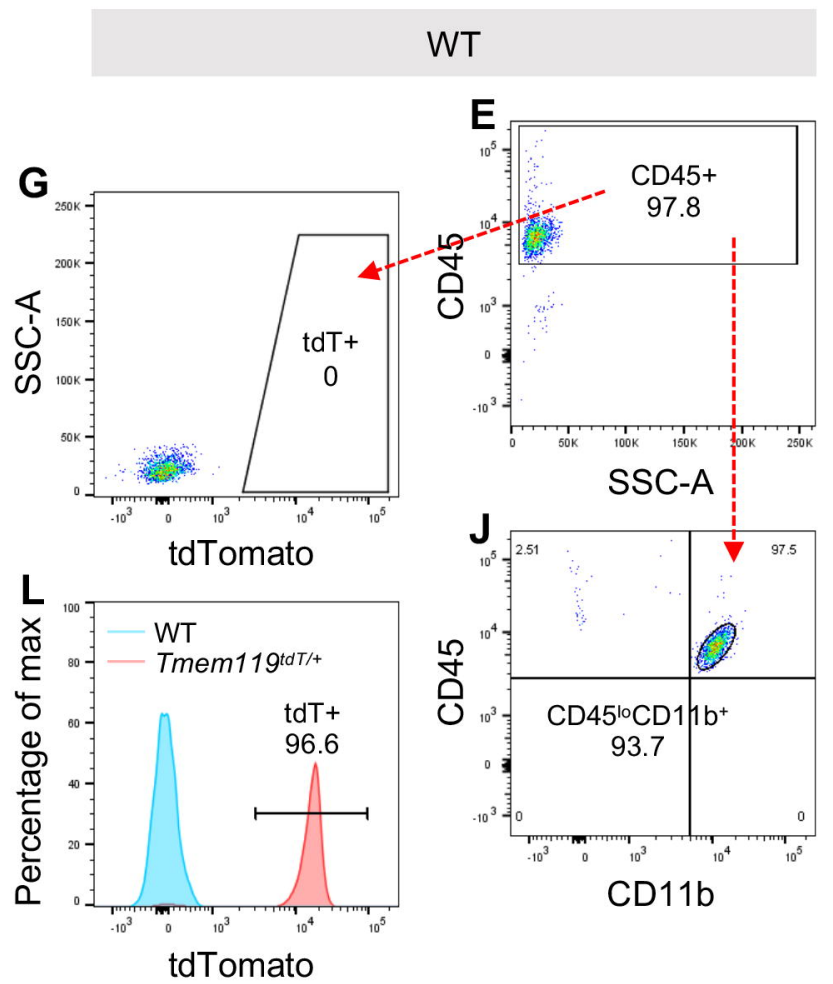

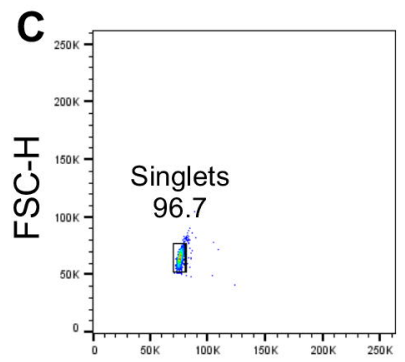

FSC-W

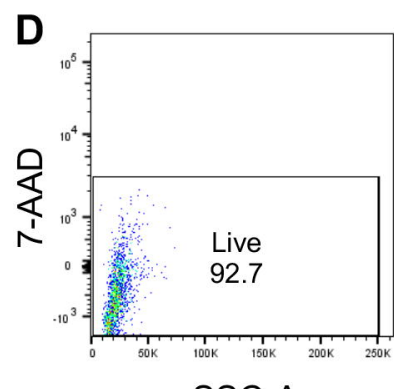

SSC-A

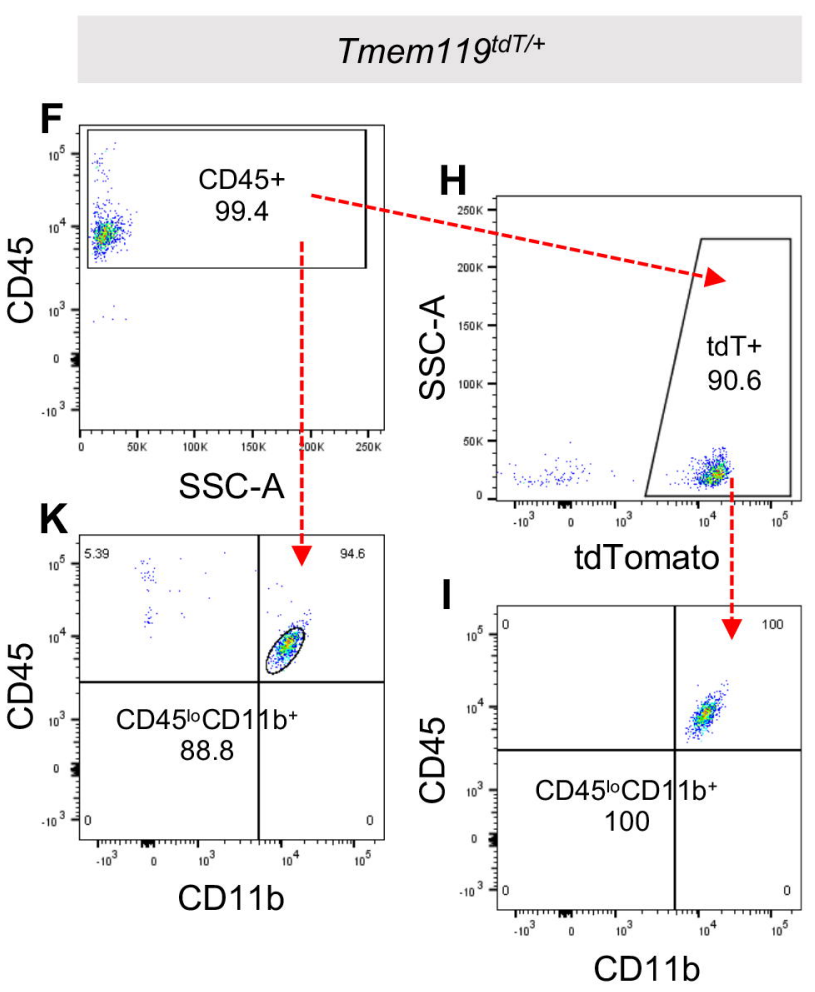




\section{Figure 6}
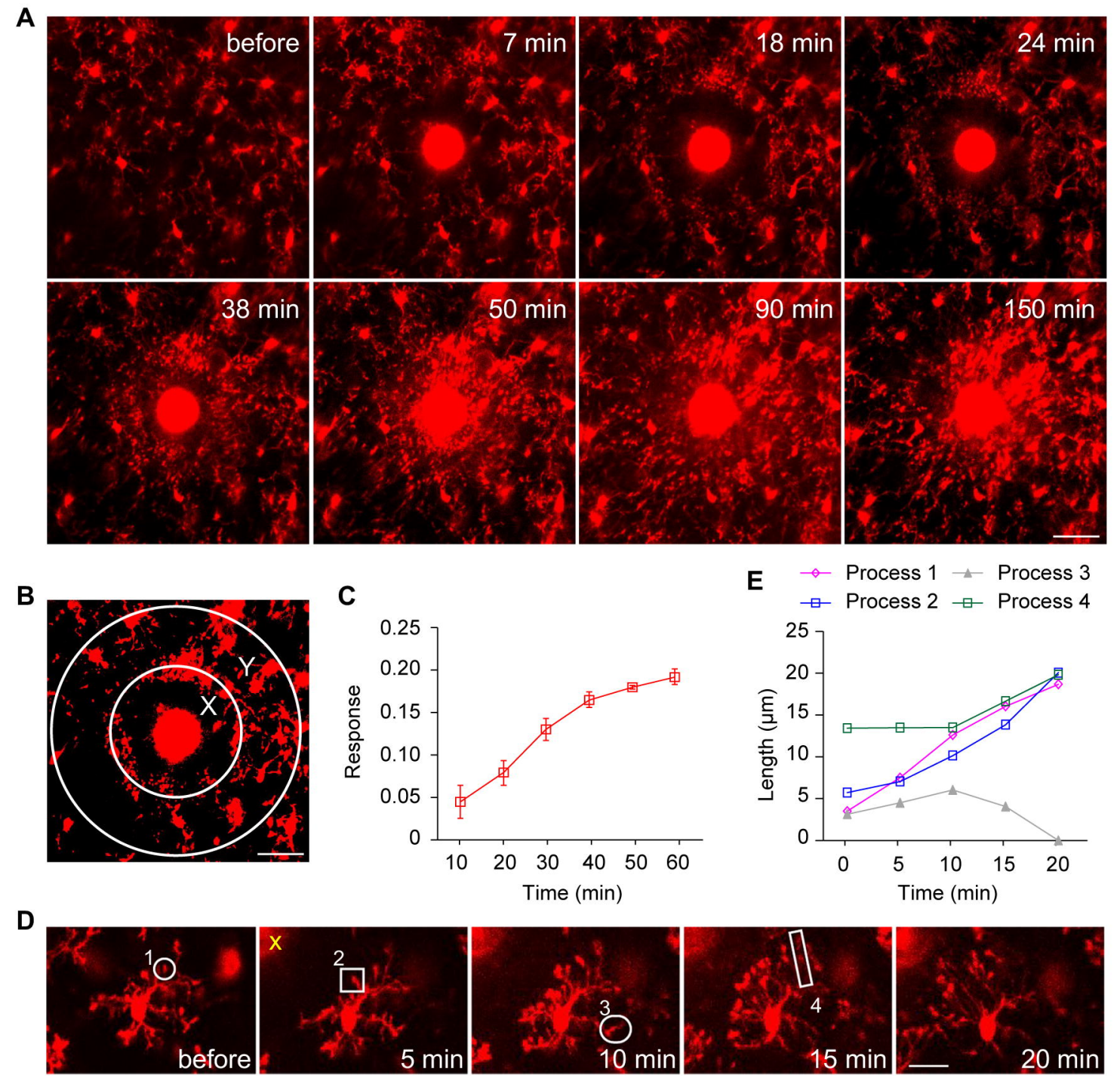